\title{
System Design of English Test Examination Room Arrangement
}

\author{
Rui Li \\ College of Foreign Languages, Bohai University, Jinzhou, 121013, China \\ selinalr@163.com
}

Keywords: examination room arrangement; English test; architecture design; functional design

\begin{abstract}
With the rapid development of the computer and universal application, its high quality and efficient work efficiency provides people with modern tools and instruments, examination room arrangement problem is a problem that has been plagued examination affairs, science and efficiently arranging examination room has become an important issue for the current development of information technology. So the content of this paper is important. Firstly, this paper studies the examination room arrangement algorithms, including the basic principles and specific algorithm process; secondly, studies the architecture design of English test examination room arrangement system, including system design principles; finally, studies the specific functional design of the system. Using the computer technology to arrange examination room for English Test, not only improved the effectiveness of examination room arrangement, but also can legitimately assign teachers and school resources, making the test more scientific and reasonable.
\end{abstract}

\section{Introduction}

National English Test is a non-degree certificate exam approved by the country, designed and developed by examination center and targeted to all citizens, the exam is to test the candidate's level of English communicative competence. It uses test to promote learning, to promote and popularize the English knowledge for the community, and to provide an objective, fair, uniform and scientific test results for English learners and employers, after years of development, it has become increasingly popular English examination [1]. With the growing number of examinee, the labor intensity of test management work is naturally increasing, to strengthen and standardize the management of examinations, effectively reducing the workload of the examination affairs staff, and reasonably arranging examination room, this paper studies English test examination room arrangement algorithm and designs the examination room arrangement system.

Examination room arrangement problem is a challenge plagued examination affairs, there are several drawbacks existed in the artificial arrangements examination room work: (1) a large amount of inputting information and tasks to be accomplished, duplication of efforts; (2) proctored teachers, subjects, examinee list, examination room and examination time and other information are enormous, complex data relationships and low effectiveness of manual arrangement; (3) in the course of examination room arrangement need to consider multi-aspect, many factors and prone to conflict; (4) the individual adjustments will take to very complex changes, largely increase the workload; (5) after the completion of examination room arrangement need a variety of hand-made reports and more complicated; (6) presence the case that appears simple repetitive tasks, increasing the amount of tasks. Therefore, science and efficiently arranging examination room has become an important issue for the current development of information technology [2].

\section{Public English Test Introduction}

The purpose of establishing PETS is to provide a scientific and reasonable evaluation criterion to improve the original English education examination. Change the passive situation that the current English exam too closed and out of touch with social needs, to provide a English exam system facing the public for the community, to promote the popularization and improvement of English in the country to adapt to the situation that China's reform and opening up and expanding foreign relations. By testing the candidate's level and awarding English grade certificate meet the needs of 
identifying English ability and the job market in society. Conducted a comprehensive test for Candidates' listening, speaking, reading, writing and other ability, promoted English teaching reform, to reverse the unfavourable situation that "do not understand, could not speak, it is difficult to communicate directly with foreigners,". Multi-level English exam system also meets the trend of lifelong learning and lifelong education in today's society [3].

PETS1 is the initial stage, English in line with the work such as taxi drivers, hotel porters, janitors, traffic police, as well as the other work in the same levels of basic needs in foreign relations; PETS2 is in the lower level, the English level basically meet requirements to enter colleges and universities continuously learning, but also in line with such hotel desk clerk, the general staff of the bank, foreign companies the general staff, as well as the other work in the same levels of basic needs in foreign relations; PETS3 is the middle-class, English has reached the requirements that non-English majors graduate level in self-study examination or non-English majors graduate in college and university, in line with enterprises and institutions executive secretary, assistant manager job, junior science and technology personnel, foreign staff, as well as the other work in the same levels of basic needs in foreign relations; PETS4 is the higher level, English level basically meet the needs of pursuing institutions of higher learning non-English majors postgraduate, in line with the basic requirements of the general professional and technical personnel or research staff, modern enterprise managers and other work; PETS5 is the most advanced, the English basically meet the needs that pursue postgraduate study for non-English majors in foreign or engaged in academic research work. The level of English is also able to meet their basic needs in professional and managerial work at home and abroad.

\section{Examination Room Arrangement Algorithm}

Registration work and examination room arrangement settings of English Test are completed independently by the provinces and municipalities. When provinces arrange examination room, they are bounded by the region, each region set up multiple test area, and set up a number of test sites in each test area, each test site is also divided into more than one examination room. The purpose of examination room arrangement is to disperse randomly and uniformly all kinds of test personnel in each test area to examination room in corresponding test area and test sites exam, in order to make the possibility that the before and after examinees belong to the same adjacent units as small as possible.

Examination room arrangement algorithm described in this article is random algorithm, random algorithm uses a random number generator or random number generation algorithm to generate a random number for each examinee, and then sorts the random numbers, finally according to random number generate examination room number and seat number for each examinee, the specific flow chart is shown in Fig. 1.

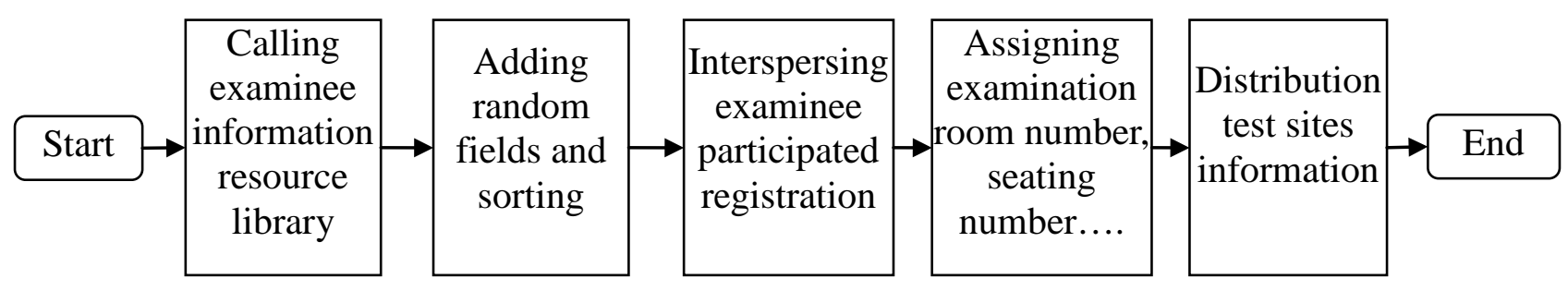

Fig. 1. Random algorithm flow diagram

Typically, the random algorithm solving the problem $\mathrm{P}$ defines as follows: Let $\mathrm{I}$ is an instance of the problem of $\mathrm{P}$, when using algorithm to solve I randomly select an input $b \in I$, according $\mathrm{b}$ to decide next algorithm, random algorithm injects randomness to algorithm, improved the flexibility of algorithm design and analysis and increased the problem-solving ability of the algorithm [4]. In the examination room arrangement, random algorithm involves a number of factors, and there are some interaction or restrict link between these basic elements. When designing algorithms firstly considers the contact between them, and secondly reflects the superiority of computing that is fast and accurate. The process using random algorithms in examination room arrangement is that firstly 
called examinee information library; then added the random field, and sorted by random fields, interspersed different examinee participated registration, assigned examination room number, seating number and ticket number; finally distributed test sites. In which the algorithm to generate random numbers is linear congruential method, the formula is as follows.

$$
\begin{gathered}
x_{i+1}=a x_{i}+b(\bmod n) \\
U_{i+1}=x_{i+1} / n
\end{gathered}
$$

In which, $a$ is multiplier (constants), $b$ is incremental (constants), $x_{0}$ is seed, $n$ is mode, $0 \leq x_{i} \leq n-1$. $x_{i}$ has the nature which is similar to the independent sampled sequences in uniform random variables, it related to the selection of these parameters. How to select the constants $a, b$ and $n$ in this method are directly related to the random properties of the generated random sequence, appropriately selected $a 、 b$ and $n$ can make $x_{i}$ generate cycle, no matter what is $x_{0}$, the cycle is the same, its cycle called generator cycle, denoted $T$, if $T=n$, claimed the generator have the full cycle and increased $n$ can improve the uniformity of $U_{i}$. appropriately selected $a 、 b$ and $n$ can make sure each integer exactly appear once over one cycle when $x_{\tilde{i}}$ in the $[0, n-1]$, thus ensuring uniformity[5].

\section{System Architecture Design}

General principles of English test examination room arrangement system is to effectively integrate existing examination resources and to enhance the computer-aided functions of examination room arrangement and forms management work, improve the efficiency of examination affairs. Specifically include the following: (1) Examination resource contains the test sites, examination room, examiners teachers, examination staff, examination information and a series of hardware and software resources, achieved information technology management and maintenance, and promoted effective integration of resources and maximum utilization; (2) examination room arrangement and examination forms management is a complex task that requires to co-ordination arrange various types of examination resources, with the auxiliary functions of the computer and efficiently completed the work in order to reduce the work intensity of test management staff; (3) facilitate access for test workers, the construction of information technology system is to break the existing information silos and to achieve the open sharing of information systems; (4) focus on the simplicity of the system and easy to maintain, examination room arrangement system design should meet the needs of daily operations and processes, meanwhile the network structure should be simple, clear hierarchy, easy to manage, easy to expand; (5) using advanced and mature technology establish a reliable system, the success of examination room arrangement system depends on the whether the system is reliable and practical, so the examination room arrangement system should use mature and reliable technology and the starting point of system design must be higher.

The architecture design of English test examination room arrangement system designed in this paper is mainly based on the MVC pattern, MVC (Model-View-Controller) pattern is presented in the GUI design, and make the data processing, and program input and output control separate each other and describes the communication between the different parts of the object, so that they do not have to be involved with each other of data model and method, make the program structure become clear and flexible. The emergence of J2EE-based MVC2 architecture achieved by JSP, Servlet and Java Beans makes MVC model widely used in the development of large-scale Web projects. MVC pattern consists of three parts: the model, view and the controller, respectively corresponding to the internal data, data representation and input-output control section [6]. The architecture diagram of English test examination room arrangement system is shown in Fig. 2. 


\section{System Functional Design}

In the English test, in order to overcome the problem of existing, for example, after examination room arrangement, examination workers export examination room arrangement data, and then the specific examination room arrangement, so that each examination room number in each time period corresponded to corresponding test room, then announce the examination room arrangements, or print post, or online publication, but also print ticket and issued to examinees, and displayed examination room number, etc., but no specific arrangements for the examination room. After examinees received ticket, according to exam time, the volume number, subjects, viewing examination room number or check each subject specific exam room arrangements online [7]. Therefore, this article using computer technology designed examination room arrangement system, its function modules include five major modules that are test sites management, examination room management, examiners teacher management, examination room arrangement, examination forms management.

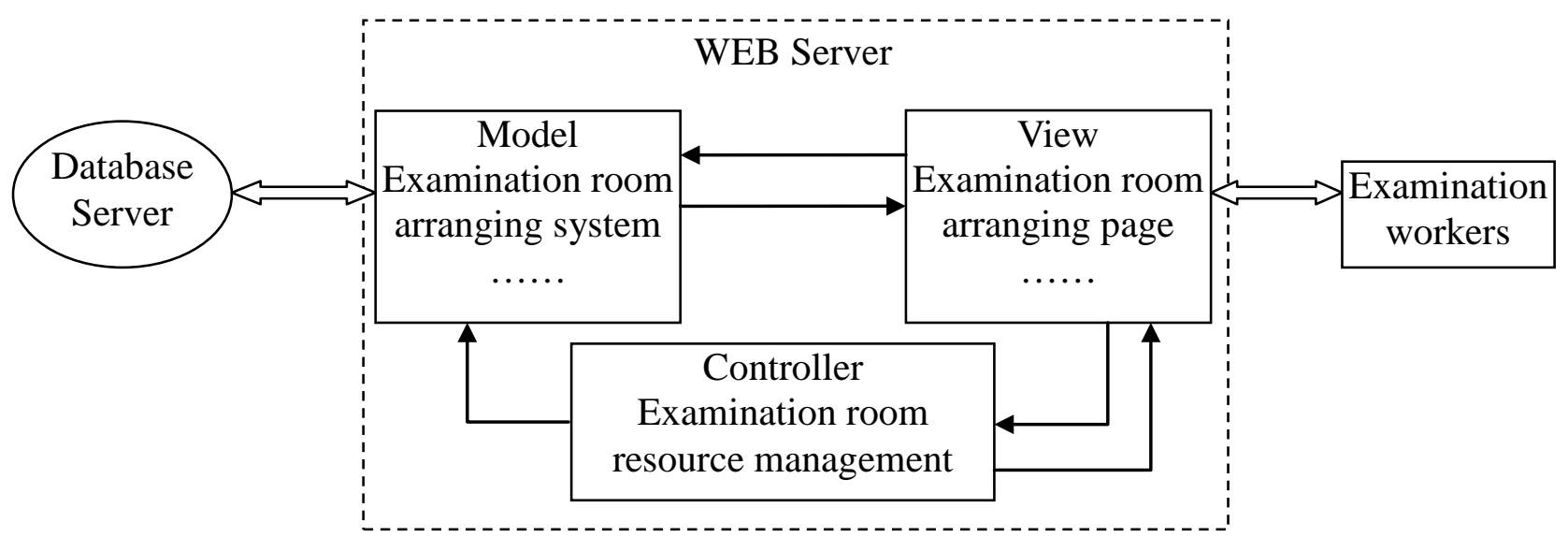

Fig. 2. Examination room arrangement system architecture diagram

Test sites management mainly includes creating, inserting, modifying, or deleting test sites; its main interface displays a list of test sites, fill test sites information via the new button to create a new test sites, it can also choose to modify existing test sites and delete operations; examination room management is that after used to select test sites, create, modify, and other operations for examination room inside the test sites, the examination room management main interface displays a list of the examination room, to create a new examination room via the new button to fill Examination room information, also through the modify button to editing existing examination room or delete examination room; proctored teacher management mainly refers to creating, inserting, modifying or deleting operation for the invigilator teachers affiliated with the selected test sites, the interface proctored teacher management main interface shows a list of examiners teachers, through the new button fill invigilator teacher information to add new examiners teacher, it can also via modify button to edit existing proctored teacher information or delete proctored teacher [8].

Examination room arrangement can be divided into written test stages and machine test phase, the principle of written stage arrangement is make the examinees participate in the same subject arrange the same examination room, easy to distribute papers and finishing work, the use of examination room followed by the order, because in the general case the examination room number and order of the classroom is corresponding, compared according to the order to use examination room with randomly assigned, one hand it can improve the utilization of the classroom, other hand it also makes the examinees participating in the same subjects get together, to facilitate the management and follow-up work, the examiners should be randomly selected to prevent cheating phenomenon; the principle of examination room arrangement in the machine test stage, choreography by the order of one to three, the time should be legitimately arranged, the order of examination room is sorted by order, the examiner teachers should be randomly selected [9]; Examination forms management illustrated the case of the generation and printing of examination 
room seating forms, management functions of other forms is same to it, the specific process is when entry into the examination forms management page, select the form type you want to generate and print, such as examination room seating list, by examination room arrangement module to get each examination room seating arrangements, including each invigilator teachers in the examination room and examinee seating information, can directly view the each examination room seating forms via Web page and print seating forms, or used the batch printing function, by the background of system make all the examination room seating list generate pdf document and sent to the client via data stream, after save it, pdf documents can be directly printed to obtain all examination room seating lists.

\section{Conclusion}

With the development of information technology, test management system as the current application system is essential, not only to get rid of relying solely on the user manual management, but also to the network direction to develop, facing the strong demand that society objectively evaluates knowledge and ability, examinations have come into being [10], especially English test. And Examination room arrangement as an important part of English test, the contents of this paper has significance for English test. English test examination room arrangement algorithm researched in this paper overcomes the big task of artificial arrangements examination room, inefficient, prone to conflict and other shortcomings, using computer technology to design English test examination room arrangement system has even more effective, and make arrangements for the examination more scientific and reasonable.

\section{Acknowledgement}

This work is supported by social science fund project of Liaoning province (L13DYY027).

\section{References}

[1] H. Lu, L. H. Long, Y. L. Zhu, "The Design and Realization of PETS Service Assisted Managing System on the Base of B/S Mode," Science Mosaic, vol. 20, no. 1, pp. 178-179, 2011.

[2] L. A. Zhang, "Shuffling Management System Design and Implementation of examination room," Master's Degree of Tianjing University, 2009.

[3] Baidu Encyclopedia, "Public English Test http://baike.baidu.com/link?url=VHq6CNcdpaOxsN3kAQ_vORHKXA5D_lMzQWfnA9pRpp Yfh7BFrQ_wBhATU117Tz-rnczle5OujGyeFDrnGNer27--CKdKRS-Y5CxKx80aUR3Y8-353 yf8R8e6FpB0X-Q0ca71XAxAYCzL66phFMXJvCdcXoNvp2URShYYyfs8x1K, 2015-8-25.

[4] H. J. Xiao, H. He, "Research and Application Strategies Randomized of Popular Algorithms," Journal of Hunan Institute of Engineering, vol. 15, no. 4, pp. 59-61, 2005.

[5] P. X. Feng, "The Algorithm Research on University Entrance Examination Seat Plumber Arranging," Master's Degree of Xinjiang Polytechnical University, 2008.

[6] F. Z. Ren, H. Zhang, S. M. Yan, F. S. Chen, "Summary of the MVC Pattern Study," Application Research of Computers, vol. 20, no. 10, pp. 1-4, 2004.

[7] M. Y. Xu, "Practice and Consideration of Appliing Database Software to Examination Room \& Seats Arrangement," Computer Programming Skills \& Maintenance, vol. 19, no. 20, pp. 135-136, 2012.

[8] J. D. L. Mi, F. Y. A. T. M. Mai, E. K. S. Ai, "Designing and Implementing Examination Room Arrangement and Job Scheduling Algorithms for Multilingual, Multi-Subject and Multi-Site 
Examination Information Management," Application Research of Computers, vol. 28, no. 5, pp. 135-136, 2012.

[9] H. X. Li, "Design and Implementation of Online Examination Arrangement Subsystem for NCRE," Master's Degree of Xi'an University of Electronic Science and Technology, 2010.

[10] H. X. Liang, "Design and Implementation of Examination Management System," Master's Degree of Xi'an Xi'an University of Technology, 2007. 